\title{
Rational periodic points for degree two polynomial morphisms on projective space
}

\author{
by
}

Benjamin Hutz (Amherst, MA)

1. Introduction and statement of results. Let $\phi: \mathbb{P}^{1} \rightarrow \mathbb{P}^{1}$ be a morphism of degree two which has a totally ramified fixed point at infinity, in other words, a polynomial morphism. We will denote by $\phi^{n}$ the $n$th iterate of $\phi$. A point $P \in K$ is called periodic of period $n$ for $\phi$ if there is a positive integer $n$ such that $\phi^{n}(P)=P$. If $n$ is the smallest such integer, it is called the primitive period of $P$ for $\phi$. Northcott's theorem [5] tells us that $\phi$ can have only finitely many rational periodic points defined over a number field and, hence, the primitive periods of rational periodic points must be bounded. For $n=1,2$, and 3 there are infinitely many examples of degree two polynomial maps defined over $\mathbb{Q}$ with $\mathbb{Q}$-rational periodic points with primitive period $n$. Morton [4] showed that there are no such maps with $\mathbb{Q}$ rational primitive 4-periodic points. Flynn, Poonen, and Schaefer [1] showed that there are no such maps with $\mathbb{Q}$-rational primitive 5-periodic points and made the following conjecture:

Conjecture 1. For $n \geq 4$ there is no quadratic polynomial $f \in \mathbb{Q}[x]$ with a rational periodic point with primitive period $n$.

More recently, Stoll [6] has shown conditionally that there are no degree two polynomial maps with $\mathbb{Q}$-rational primitive 6-periodic points. For degree two rational maps, Manes [3, Theorem 4] showed the existence of maps with $\mathbb{Q}$-rational periodic points of primitive period 4 and provided evidence for there being no maps with $\mathbb{Q}$-rational points of primitive period 5 or 6 . This article examines the possible primitive period of a $\mathbb{Q}$-rational periodic point for a degree two polynomial morphism on $\mathbb{P}^{N}$ defined over $\mathbb{Q}$. 
Definition. We define a polynomial map $\phi: \mathbb{P}^{N} \rightarrow \mathbb{P}^{N}$ of degree $d$ with coordinates $\left[x_{0}, \ldots, x_{N}\right]$ as

$$
\phi\left(x_{0}, \ldots, x_{N}\right)=\left[\phi_{0}\left(x_{0}, \ldots, x_{N}\right), \ldots, \phi_{N-1}\left(x_{0}, \ldots, x_{N}\right), x_{N}^{d}\right],
$$

where each $\phi_{i}$ is a homogeneous form of degree $d$ in the variables $x_{0}, \ldots, x_{N}$. Such a map is a morphism if $\phi_{0}, \ldots, \phi_{N-1}$ have no nontrivial common zeros when $x_{N}=0$.

1.1. A first example. Consider a degree two polynomial map $\phi: \mathbb{P}^{1} \rightarrow \mathbb{P}^{1}$ given by

$$
\phi(x, y)=\left[a x^{2}+b x y+c y^{2}, y^{2}\right] .
$$

We wish to find constants $a, b$, and $c$ such that $P=[0,1]$ is a periodic point of primitive period 3 for $\phi$. To do so we choose any two distinct other points $P_{1}$ and $P_{2}$ and solve the three linear equations $\phi(P)=P_{1}, \phi\left(P_{1}\right)=P_{2}$, and $\phi\left(P_{2}\right)=P$ in the three unknowns $a, b$, and $c$ to find a suitable map $\phi$. Since we will use a similar, albeit more complicated, construction in Theorem 1 , we explore this construction in detail for this simple example.

We see that

$$
\phi([0,1])=[c, 1],
$$

so we choose $c \neq 0$, say $c=1$. Then we have

$$
\phi([1,1])=[a+b+1,1] .
$$

We now choose $b$ so that

$$
\phi([1,1]) \neq[0,1],[1,1],
$$

say $b=1-a$. Then $\phi([1,1])=[2,1]$ and so

$$
\phi([2,1])=[2 a+3,1] .
$$

Finally, we choose $a=-3 / 2$ to have $\phi([2,1])=[0,1]$, making the degree two polynomial map

$$
\phi([x, y])=\left[-3 / 2 x^{2}+5 / 2 x+1, y^{2}\right]
$$

have $[0,1]$ as a primitive 3 -periodic point.

Trying to construct a $\mathbb{Q}$-rational primitive 4-periodic point in the same manner, at a minimum, requires more care. The obstruction lies in having to solve four equations in three unknowns. Conjecture 1 states that for a degree two polynomial morphism on $\mathbb{P}^{1}$ it is never possible to solve these larger systems of equations and the number of coefficients $\left(\begin{array}{c}1+2 \\ 2\end{array}\right)=3$ is an upper bound on the primitive $\mathbb{Q}$-rational periods.

Theorem 1 demonstrates an infinite family of polynomial maps on $\mathbb{P}^{N}$ with periodic points with primitive period larger than $\left(\begin{array}{c}N+2 \\ 2\end{array}\right)$. Theorem 2 shows that these families contain infinitely many maps which are in fact morphisms of $\mathbb{P}^{N}$. Theorem 3 uses Theorems 1 and 2 to show that the 
primitive period of $\mathbb{Q}$-rational periodic points for polynomial morphisms of $\mathbb{P}^{N}$ grows faster than $c(k) N^{k}$ for any $k$ and some constant $c(k)$.

1.2. The main results. In general, we can construct a degree two polynomial map with a $\mathbb{Q}$-rational periodic point with primitive period equal to the number of coefficients of a quadratic form in $N+1$ variables $\left(\begin{array}{c}N+2 \\ 2\end{array}\right)=$ $(N+1)(N+2) / 2$, by choosing one coefficient with each successive iterate as we did above for $\mathbb{P}^{1}$. We show that for $N \geq 2$ we can construct polynomial maps on $\mathbb{P}^{N}$ with a periodic point with primitive period larger than this value.

Theorem 1. Let $N \geq 2$. There is an infinite family of degree two polynomial maps $\phi: \mathbb{P}^{N} \rightarrow \mathbb{P}^{N}$ with a $\mathbb{Q}$-rational periodic point with primitive period

$$
\leq \begin{cases}7=(N+1)(N+2) / 2+1 & \text { for } N=2, \\ (N+1)(N+2) / 2+\lfloor(N-1) / 2\rfloor & \text { for } N \geq 3,\end{cases}
$$

where $\lfloor x\rfloor$ denotes the greatest integer less than or equal to $x$. Moreover, the dimension of the family is at least $N$.

THEOREM 2. The infinite family of maps constructed in Theorem 1 contains infinitely many morphisms.

TheOREM 3. For $N$ large enough, there exists a degree two polynomial morphism of $\mathbb{P}^{N}$ with a $\mathbb{Q}$-rational periodic point with primitive period larger than $c(k) N^{k}$ for any $k$ and some constant $c(k)$ depending on $k$.

In general, the bounds in Theorem 1 are not upper bounds on the primitive period. Several examples of polynomial morphisms with $\mathbb{Q}$-rational points with larger primitive period are included at the end of the article.

2. Proof of Theorem 1. We denote the $i$ th coordinate of a point $P \in \mathbb{P}^{N}$ as $x_{i}(P)$, and define the polynomial map $\phi: \mathbb{P}^{N} \rightarrow \mathbb{P}^{N}$ by

$$
x_{i}\left(\phi\left(x_{0}, \ldots, x_{N}\right)\right)= \begin{cases}\sum_{j=0}^{N-1} \sum_{k=j}^{N} c_{i}(j, k) x_{j} x_{k} & \text { for } i=0, \ldots, N-1, \\ c_{N}(N, N) x_{N}^{2} & \text { for } i=N .\end{cases}
$$

We denote the $n$th image of $P$ by $\phi$ as $\phi^{n}(P)=P_{n}$.

The method of construction is to choose appropriate values of the constants $c_{i}(j, k)$ so that the coordinates of each iterate are linear in at most two of the $c_{i}(j, k)$. When we have chosen all of the $c_{i}(j, k)$ with $j \neq k$, we will then be able to choose $c_{i}(j, j)$ so that $\phi(P)$ is determined and $\phi(\phi(P))$ is linear in one of the $c_{i}(j, j)$, allowing the primitive period to increase beyond the trivial value $\left(\begin{array}{c}N+2 \\ 2\end{array}\right)$ determined by the number of coefficients. We treat the case of $N=2$ separately.

In Lemma 1, we choose the initial sequence of images by specifying $c_{i}(j, k), 0 \leq i \leq N$, for one pair $(j, k)$ for each image. Then we proceed 
with the construction to increase the primitive period beyond the trivial lower bound.

Lemma 1. Let $\phi: \mathbb{P}^{N} \rightarrow \mathbb{P}^{N}$ be a degree two polynomial map. We may choose the first $\left(N^{2}+N\right) / 2-1$ images of $[0, \ldots, 0,1]$ as

$$
\begin{aligned}
& {[0, \ldots, 0,1] \stackrel{\phi}{\rightarrow}[1,0, \ldots, 0,1] \stackrel{\phi}{\rightarrow}[0,1,0, \ldots, 0,1] \stackrel{\phi}{\rightarrow} \ldots \stackrel{\phi}{\rightarrow}[0, \ldots, 0,1,1]} \\
& \quad \stackrel{\phi}{\rightarrow}[1,1,0, \ldots, 0,1] \stackrel{\phi}{\rightarrow}[0,1,1,0, \ldots, 0,1] \stackrel{\phi}{\rightarrow} \ldots \stackrel{\phi}{\rightarrow}[0, \ldots, 0,1,1,1] \\
& \quad \stackrel{\phi}{\rightarrow}[1,1,1,0, \ldots, 0,1] \stackrel{\phi}{\rightarrow}[0,1,1,1,0, \ldots, 0,1] \stackrel{\phi}{\rightarrow} \ldots \stackrel{\phi}{\rightarrow}[0, \ldots, 0,1,1,1,1] \\
& \quad \vdots \\
& \quad \stackrel{\phi}{\rightarrow}[1, \ldots, 1,0,1] \stackrel{\phi}{\rightarrow}[0,1, \ldots, 1,1] \stackrel{\phi}{\rightarrow}[1, \ldots, 1]
\end{aligned}
$$

by choosing all of the $c_{i}(j, k)$ except $c_{i}(0, N-1)$ and $c_{i}(k, k)$ for each $0 \leq$ $k \leq N-1$ and each $0 \leq i \leq N-1$. Furthermore, $x_{i}(\phi([1, \ldots, 1]))$ is of the form

$$
a_{i} c_{i}(0, N-1)+b_{i}
$$

for some constants $a_{i}, b_{i}$ for all $0 \leq i \leq N-1$.

Proof. Let $P=[0, \ldots, 0,1]$. Then $x_{i}(\phi(P))$ is linear in $c_{i}(N, N)$, so we can choose

$$
\phi(P)=[1,0, \ldots, 0,1]
$$

by setting

$$
c_{i}(N, N)= \begin{cases}1 & \text { for } i=0 \text { and } i=N \\ 0 & \text { for } 1 \leq i \leq N-1\end{cases}
$$

Next we choose the sequence of points

$$
\begin{aligned}
{[1,0, \ldots, 0,1] \stackrel{\phi}{\rightarrow}[0,1,0, \ldots, 0,1] \stackrel{\phi}{\rightarrow} \cdots } \\
\stackrel{\phi}{\rightarrow}[0,0, \ldots, 0,1,1] \stackrel{\phi}{\rightarrow}[1,1,0, \ldots, 0,1],
\end{aligned}
$$

where

$$
[1,1,0, \ldots, 0,1]=P_{N+1} .
$$

We can do this because $x_{i}\left(\phi\left(P_{j}\right)\right)$ is of the form

$$
x_{i}\left(\phi\left(P_{j}\right)\right)= \begin{cases}c_{i}(j-1, j-1)+c_{i}(j-1, N)+1 & \text { for } i=0 \\ c_{i}(j-1, j-1)+c_{i}(j-1, N) & \text { for } 1 \leq i \leq N-1, \\ 1 & \text { for } i=N .\end{cases}
$$

We choose

$$
c_{i}(j-1, N)= \begin{cases}-1-c_{i}(j-1, j-1) & \text { for } i=0, \\ 1-c_{i}(j-1, j-1) & \text { for } i=j-1, \\ -c_{i}(j-1, j-1) & \text { otherwise. }\end{cases}
$$


REmark. With these choices of $c_{i}(N, N)$ and $c_{i}(k, N)$ for all $0 \leq k \leq$ $N-1$, the image $x_{k}\left(\phi\left(x_{0}, x_{1}, \ldots, x_{N-1}, 1\right)\right)$ contains terms of the form $c_{k}(i, i) x_{i}^{2}-c_{k}(i, i) x_{i}$ for all $0 \leq i \leq N$ and $0 \leq k<N-1$. Consequently, if $x_{i}=1$ or $x_{i}=0$, then $c_{k}(i, i)$ does not appear in the $k$ th coordinate of the image.

Next we choose the sequence of images

$$
\begin{aligned}
{[1,1,0, \ldots, 0,0,1] } & \stackrel{\phi}{\rightarrow}[0,1,1,0, \ldots, 0,1] \stackrel{\phi}{\rightarrow} \ldots \\
& \stackrel{\phi}{\rightarrow}[0, \ldots, 0,1,1,1] \stackrel{\phi}{\rightarrow}[1,1,1,0, \ldots, 0,1]
\end{aligned}
$$

until

$$
P_{2 N-1}=[1,1,1,0, \ldots, 0,1] .
$$

We can do this because we have already chosen $c_{i}(k, N)$ for all $0 \leq k \leq N-1$ and $c_{i}(N, N)$, causing the $i$ th coordinate for all $0 \leq i \leq N-1$ of each image in this sequence to be linear only in the single coefficient $c_{i}(k, k+1)$ for some $0 \leq k \leq N-2$.

Next we choose the sequence of images

$$
\begin{aligned}
& {[1,1,1,0, \ldots, 0,1] \stackrel{\phi}{\rightarrow}[0,1,1,1,0, \ldots, 0,1] \stackrel{\phi}{\rightarrow} \cdots } \\
& \stackrel{\phi}{\rightarrow}[0, \ldots, 0,1,1,1,1] \stackrel{\phi}{\rightarrow}[1,1,1,1,0, \ldots, 0,1] .
\end{aligned}
$$

Since we have already chosen $c_{i}(N, N), c_{i}(k, N)$ for all $0 \leq k \leq N-1$, and $c_{i}(k, k+1)$ for all $0 \leq k \leq N-2$ and $0 \leq i \leq N-1$, the $i$ th coordinate of these iterates is linear in $c_{i}(k, k+2)$ for some $0 \leq k \leq N-3$.

We repeat this process until we have

$$
\begin{aligned}
& \phi\left(P_{\left(N^{2}+N\right) / 2-3}\right)=[1, \ldots, 1,0,1,1] \quad\left(\text { linear in } c_{i}(0, N-2)\right), \\
& \left.\phi\left(P_{\left(N^{2}+N\right) / 2-2}\right)=[0,1, \ldots, 1] \quad \text { (linear in } c_{i}(1, N-1)\right) \text {, } \\
& \phi\left(P_{\left(N^{2}+N\right) / 2-1}\right)=[1, \ldots, 1] \quad\left(\text { linear in } c_{i}(0, N-1)\right) .
\end{aligned}
$$

The only coefficients not yet chosen are $c_{j}(i, i)$ for all $0 \leq i \leq N-1$ and $c_{j}(0, N-1)$. We also know that $x_{i}(\phi(P))$ is linear in $c_{i}(0, N-1)$ for all $0 \leq i \leq N-1$.

We are now ready to increase the primitive period beyond the trivial lower bound.

Proof of Theorem 1 .

CASE 1: $N=2$. We begin where Lemma 1 finished. We have chosen $c_{i}(2,2)$ for all $0 \leq i \leq 2$ and $c_{i}(0,2)$ and $c_{i}(1,2)$ for all $0 \leq i \leq 1$ to have the sequence of points

$$
[0,0,1] \stackrel{\phi}{\rightarrow}[1,0,1] \stackrel{\phi}{\rightarrow}[0,1,1] \stackrel{\phi}{\rightarrow}[1,1,1]
$$


Now we choose $c_{i}(0, N-1)=c_{i}(0,1)$ so that

$$
\phi([1,1,1])=[0, K(0,1), 1]
$$

for some constant $K(0,1) \notin\{0,1\}$. Each coordinate $x_{i}(\phi([0, K(0,1), 1]))$ is linear in $c_{i}(1,1)$. We then choose the $c_{i}(1,1)$ so that

$$
\phi([0, K(0,1), 1])=[0, K(1,1), 1],
$$

where $K(1,1)$ is a constant with $K(1,1) \notin\{0,1, K(0,1)\}$. So we have three points chosen of the form $\left[0, x_{1}, 1\right]$ whose images are given by

$$
\begin{gathered}
{[0,0,1] \stackrel{\phi}{\rightarrow}[1,0,1],} \\
{[0,1,1] \stackrel{\phi}{\rightarrow}[1,1,1],} \\
{[0, K(0,1), 1] \stackrel{\phi}{\rightarrow}[0, K(1,1), 1] .}
\end{gathered}
$$

Since $x_{i}\left(\phi\left[0, x_{1}, 1\right]\right)$ is a quadratic polynomial in $x_{1}$ for $0 \leq i \leq 1$, the image $\phi([0, K(1,1), 1])$ is determined by these three known points and is of the form

$$
\phi([0, K(1,1), 1])=\left[k_{0}, k_{1}, 1\right]
$$

for some constants $k_{0}$ and $k_{1}$. Note that we may need to exclude finitely many choices of $K(0,1)$ and $K(1,1)$ (and hence of $c_{1}(0,1)$ and $c_{1}(1,1)$ ) so that $k_{0} \notin\{0,1\}$. Therefore each $x_{i}\left(\phi\left(\left[k_{0}, k_{1}, 1\right]\right)\right)$ is linear in $c_{i}(0,0)$ and we choose the $c_{i}(0,0)$ so that

$$
\phi(\phi([0, K(1,1), 1]))=[0,0,1] .
$$

This is a primitive 7-periodic point, and the family is dimension 2 since we can choose $c_{1}(0,1)$ and $c_{1}(1,1)$ arbitrarily (with finitely many exceptions).

It is easy to modify this construction to get points with primitive periods $1, \ldots, 6$ because at each stage we are linear in at most two variables. So we simply choose the constant so that $\phi^{n}(P)=[0,0,1]$ at the appropriate iterate. The dimension of these families is larger since there are more free coefficients.

CASE 2: $N \geq 3$. We begin where Lemma 1 finished and choose the $c_{i}(0, N-1)$ so that

$$
\phi\left(P_{\left(N^{2}+N\right) / 2}\right)=[0, K(0, N-1), 1, \ldots, 1]
$$

where $K(0, N-1) \notin\{0,1\}$ is a constant. Each coordinate $x_{i}(\phi([0, K(0, N-1)$, $1, \ldots, 1]))$ is linear in $c_{i}(1,1)$. Choose the $c_{i}(1,1)$ to have

$$
\phi([0, K(0, N-1), 1, \ldots, 1])=[K(1,1), 1, \ldots, 1,0,1],
$$

where $K(1,1) \notin\{0,1\}$ is some constant. Now the $i$ th coordinate of the image of $[K(1,1), 1, \ldots, 1,0,1]$ is linear in $c_{i}(0,0)$ for all $0 \leq i \leq N-1$. So 
we choose the $c_{i}(0,0)$ so that

$$
\phi([K(1,1), 1, \ldots, 1,0,1])=[0, K(0,0), 1, \ldots, 1]
$$

for some constant $K(0,0) \notin\{0,1, K(0, N-1)\}$. Note that there are three points of the form $\left[0, x_{1}, 1, \ldots, 1\right]$ whose images are given by

$$
\begin{aligned}
& {[0,1,1, \ldots, 1] } \stackrel{\phi}{\rightarrow}[1, \ldots, 1], \\
& {[0, K(0, N-2), 1, \ldots, 1] } \stackrel{\phi}{\rightarrow}[K(1,1), 1, \ldots, 1,0,1], \\
& {[0,0,1, \ldots, 1] \stackrel{\phi}{\rightarrow}[1,1, \ldots, 1,0,1] . }
\end{aligned}
$$

Since each $x_{i}\left(\phi\left(\left[0, x_{1}, 1, \ldots, 1\right]\right)\right)$ is a degree two polynomial in $x_{1}$, the three known points and their images completely determine the image of any point of the form $\left[0, x_{1}, 1, \ldots, 1\right]$. The 0 th coordinate is a nonconstant function of $x_{1}$ and the $(N-1)$ st coordinate is a nonconstant function of $x_{1}$. The remaining coordinates take on a constant value of 1 . Therefore, we have

$$
\phi([0, K(0,0), 1, \ldots, 1])=\left[k_{0}, 1,1, \ldots, 1, k_{N-1}, 1\right]
$$

for some constants $k_{0}$ and $k_{N-1}$ with $k_{N-1} \notin\{0,1\}$ (again we may need to exclude finitely many choices of $K(1,1)$ and $K(0, N-2)$ so that $k_{N-1} \notin$ $\{0,1\})$. In particular, since the $c_{i}(0,0)$ are already chosen, each coordinate $x_{i}\left(\phi\left(\left[k_{0}, 1,1, \ldots, 1, k_{N-1}, 1\right]\right)\right)$ is linear in $c_{i}(N-1, N-1)$. From our choice of $[0, K(0,0), 1, \ldots, 1]$ we have determined $\phi([0, K(0,0), 1, \ldots, 1])$ and have $\phi(\phi([0, K(0,0), 1, \ldots, 1]))$ as our next iterate to consider. We have thus increased the primitive period of $[0, \ldots, 0,1]$ by two with the choice of the $c_{i}(0,0)$.

If $N=3$ we are done since we are linear in the $c_{i}(N-1, N-1)$ and they are the only unchosen coefficients; so we choose the $c_{i}(N-1, N-1)$ to make the point periodic.

For $N>3$ we repeat the process. Choose the $c_{i}(N-1, N-1)$ to get

$$
[0,0, K(N-1, N-1), 1, \ldots, 1,0,1]
$$

with $K(N-1, N-1) \notin\{0,1\}$. The coordinates of the image are linear in the $c_{i}(2,2)$ so we choose

$$
\phi([0,0, K(N-1, N-1), 1, \ldots, 1,0,1])=[0,0, K(2,2), 1, \ldots, 1,0,1],
$$

for $K(2,2) \notin\{0,1, K(N-1, N-1)\}$. Then $\phi([0,0, K(2,2), 1, \ldots, 1,0,1])$ is completely determined since we have three points of the form $\left[0,0, x_{2}\right.$, $1, \ldots, 1,0,1]$ whose images are known. These images are

$$
\begin{aligned}
& {[0,0, K(N-1, N-1), 1, \ldots, 1,0,1] } \stackrel{\phi}{\rightarrow}[0,0, K(2,2), 1, \ldots, 1,0,1], \\
& {[0,0,1,1, \ldots, 1,0,1] \stackrel{\phi}{\rightarrow}[0,0,0,1, \ldots, 1,1,1,1], } \\
& {[0,0,0,1, \ldots, 1,0,1] \stackrel{\phi}{\rightarrow}[0,0,0,0,1, \ldots, 1,1,1,1] . }
\end{aligned}
$$


We have

$$
\phi([0,0, K(2,2), 1, \ldots, 1,0,1])=[0,0, x, y, 1, \ldots, 1, z, 1]
$$

for some constants $x, y$, and $z$. Note that we may need to exclude finitely many choices of $K(N-1, N-1)$ and $K(2,2)$ so that $y \notin\{0,1\}$. We have already chosen the $c_{i}(2,2)$ and the $c_{i}(N-1, N-1)$, so each coordinate $x_{i}(\phi(\phi([0,0, K(2,2), 1, \ldots, 1,0,1])))$ is linear in $c_{i}(3,3)$, again increasing the primitive period by 2 with the choice of a single set of coefficients $c_{i}(2,2)$.

Continuing in this manner, we choose the $c_{i}(k, k)$ to get

$$
[0, \ldots, 0, K(k, k), 1, \ldots, 1,0,1],
$$

where $K(k, k) \notin\{0,1\}$ and is the $(k+1)$ st coordinate. The $i$ th coordinate of the image is linear in $c_{i}(k+1, k+1)$. We choose $\phi([0, \ldots, 0, K(k, k), 1, \ldots, 1,0,1])=[0, \ldots, 0, K(k+1, k+1), 1, \ldots, 1,0,1]$, where $K(k+1, k+1)$ is the $(k+1)$ st coordinate and $K(k+1, k+1) \notin$ $\{0,1, K(k, k)\}$. The image $\phi([0, \ldots, 0, K(k+1, k+1), 1, \ldots, 1,0,1])$ is completely determined since we have three points of the form $\left[0, \ldots, 0, x_{k+1}\right.$, $1, \ldots, 1,0,1]$ whose images are known; they are

$$
\begin{gathered}
{[0, \ldots, 0, K(k, k), 1, \ldots, 1,0,1] \stackrel{\phi}{\rightarrow}[0, \ldots, 0, K(k+1, k+1), 1, \ldots, 1,0,1],} \\
{[0, \ldots, 0,1,1, \ldots, 1,0,1] \stackrel{\phi}{\rightarrow}[0, \ldots, 0,0,1, \ldots, 1,1,1,1],} \\
{[0, \ldots, 0,0,1, \ldots, 1,0,1] \stackrel{\phi}{\rightarrow}[0, \ldots, 0,0,0,1, \ldots, 1,1,1,1] .}
\end{gathered}
$$

We have

$$
\phi([0, \ldots, 0, K(k+1, k+1), 1, \ldots, 1,0,1])=[0, \ldots, 0, x, y, 1, \ldots, 1, z, 1]
$$

for some constants $x, y$, and $z$. Note that we may need to exclude finitely many choices of $K(k, k)$ and $K(k+1, k+1)$ so that $y \notin\{0,1\}$. We have already chosen the $c_{i}(k+1, k+1)$ and the $c_{i}(N-1, N-1)$ so each $x_{i}(\phi(\phi([0, \ldots, 0$, $K(k+1, k+1), 1, \ldots, 1,0,1])))$ is linear in $c_{i}(k+2, k+2)$, again increasing the primitive period by 2 .

We continue this process until the only unchosen coefficients are either $\left\{c_{i}(N-2, N-2), c_{i}(N-3, N-3)\right\}$ or $\left\{c_{i}(N-2, N-2)\right\}$. In this first case, we do not have enough unchosen coefficients remaining to increase the primitive period further beyond the trivial value, so we simply choose the $c_{i}(N-3, N-3)$ to have the point

$$
[0, \ldots, 0, K(N-3, N-3), 1,1]
$$

with $K(N-3, N-3) \notin\{0,1\}$. Each $x_{i}(\phi([0, \ldots, 0, K(N-3, N-3), 1,1]))$ is linear in $c_{i}(N-2, N-2)$. We have now reduced to the second case and choose the $c_{i}(N-2, N-2)$ to have

$$
\phi([0, \ldots, 0, K(N-3, N-3), 1,1])=[0,0, \ldots, 0,1],
$$

making the point periodic of primitive period $(N+1)(N+2) / 2+\lfloor(N-1) / 2\rfloor$. 
Note that along the way we were able to choose

$$
\left\{c_{1}(0, N-1), c_{1}(0,0), c_{0}(1,1), \ldots, c_{N-2}(N-3, N-3), c_{2}(N-1, N-1)\right\}
$$

arbitrarily, except for excluding a finite set of values, making this an infinite family of dimension $N$.

It is easy to modify this construction to get points with periods $<$ $(N+1)(N+2) / 2+\lfloor(N-1) / 2\rfloor$ since at each stage we are linear in at most two variables. So we simply choose the coefficients so that $\phi^{n}(P)=$ $[0, \ldots, 0,1]$ at the appropriate iterate. The dimension of these families is larger since there are more free coefficients.

3. Proof of Theorem 2, We will use the theory of Macaulay resultants to show that we can choose the coefficients of the maps in Theorem 1 so that they are morphisms; in other words, so that $\phi_{0}, \ldots, \phi_{N}$ have no nontrivial common zeros. Following [2] given $N+1$ homogeneous forms $F_{0}, \ldots, F_{N}$ of degree $d_{i}$ in $N+1$ variables $x_{0}, \ldots, x_{N}$, construct a matrix denoted $M_{d}\left(F_{0}, \ldots, F_{N}\right)$ where $d=1+\sum_{i}\left(d_{i}-1\right)$. The columns of $M_{d}$ correspond to the monomials of degree $d$ in the variables $x_{0}, \ldots, x_{N}$, and the rows correspond to polynomials of the form $r F_{i}$ where $r$ is a monomial such that $\operatorname{deg}\left(r F_{i}\right)=d$. The entries of $M_{d}$ are the coefficients of the column monomials in the row polynomials. The matrix has $\left(\begin{array}{c}N+d \\ d\end{array}\right)$ columns and the number of rows corresponding to each $F_{i}$ is $\left(\begin{array}{c}N+d-d_{i} \\ d-d_{i}\end{array}\right)$. It is the transpose of the matrix of the linear map

$$
\left(P_{0}, \ldots, P_{N}\right) \mapsto P_{0} F_{0}+\cdots+P_{N} F_{N},
$$

where $P_{i}$ is homogeneous of degree $d-d_{i}$. Consider the maximal minors of $M_{d}\left(F_{0}, \ldots, F_{N}\right)$. The determinants of these minors are polynomials in the coefficients of $F_{0}, \ldots, F_{N}$. Let $R$ be the greatest common divisor of these determinants (as polynomials in the coefficients). Then $R$ is called the resultant of $F_{0}, \ldots, F_{N}$ and (among other properties) it satisfies $R=0$ if and only if the forms $F_{0}, \ldots, F_{N}$ have a common nontrivial zero.

Proof of Theorem 2. We are in the case of $N+1$ homogeneous forms $\phi_{i}$ in $N+1$ variables $x_{0}, \ldots, x_{N}$. We have each $\phi_{i}$ of degree 2 and hence $d=N+2$. We will show that the Macaulay matrix has a maximal minor that has nonzero determinant and hence that the resultant is nonzero for infinitely many maps in the family. In the matrix there are $\left(\begin{array}{c}2 N+2 \\ N+2\end{array}\right)$ columns corresponding to all of the monomials with degree $N+2$ and $(N+1)\left(\begin{array}{c}2 N \\ N\end{array}\right)$ rows corresponding to the $\left(\begin{array}{c}2 N \\ N\end{array}\right)$ monomials of degree $d-2$ for each of the $N+1$ forms $\phi_{i}$. We need to extract a $\left(\begin{array}{c}2 N+2 \\ N+2\end{array}\right) \times\left(\begin{array}{c}2 N+2 \\ N+2\end{array}\right)$ minor with nonzero determinant. We first consider the case of largest possible period from Theorem 1. 
For $N=2$ we can write down the matrix (but do not do so here) and explicitly check that it has a maximal minor with nonzero determinant.

For $N \geq 3$ define (with $N-2$ replaced with $N-1$ for $N=3$ )

$$
\begin{aligned}
S_{N} & =\left\{F: \operatorname{deg}(F)=d, x_{N}^{2} \mid F\right\}, \\
S_{N-2} & =\left\{F: \operatorname{deg}(F)=d, x_{N}^{2} \nmid F, \text { and } x_{N-2}^{2} \mid F\right\}, \\
S_{N-1} & =\left\{F: \operatorname{deg}(F)=d, x_{N}^{2} \nmid F, x_{N-2}^{2} \nmid F, \text { and } x_{N-1}^{2} \mid F\right\}, \\
S_{N-3} & =\left\{F: \operatorname{deg}(F)=d, x_{N}^{2} \nmid F, x_{N-1}^{2} \nmid F, x_{N-2}^{2} \nmid F, \text { and } x_{N-3}^{2} \mid F\right\}, \\
S_{N-4} & =\left\{F: \operatorname{deg}(F)=d, x_{N}^{2} \nmid F, \ldots, x_{N-3}^{2} \nmid F, \text { and } x_{N-4}^{2} \mid F\right\}, \\
& \vdots \\
S_{0} & =\left\{F: \operatorname{deg}(F)=d, x_{N}^{2} \nmid F, \ldots, x_{1}^{2} \nmid F, \text { and } x_{0}^{2} \mid F\right\} .
\end{aligned}
$$

Order the columns in reverse lexicographic order, $x_{N}>x_{N-1}>\cdots>x_{0}$, with the largest to the left. For the columns corresponding to a monomial in $S_{N}$, choose the row with a 1 on the diagonal (the row contains all 0's except one entry which is 1 since $\left.\phi_{N}\left(x_{0}, \ldots, x_{N}\right)=x_{N}^{2}\right)$. For columns corresponding to monomials in $S_{2 k}$ with $k \neq 0$, choose the row with $c_{2 k}(2 k, 2 k)$ on the diagonal. For columns corresponding to monomials in $S_{2 k-1}$ with $k \neq 1$, choose the row with $c_{2 k}(2 k-1,2 k-1)$ on the diagonal. For $S_{1}$ we choose the row with $c_{0}(1,1)$ on the diagonal, and for $S_{0}$ we choose the row with $c_{1}(0,0)$ on the diagonal. Finally, for columns corresponding to monomials in $S_{N-2}$ we fix $i>1$ odd and choose the row with $c_{i}(N-2, N-2)$ on the diagonal (use $S_{N-1}$ and $c_{i}(N-1, N-1)$ for $N=3$ ).

We have two facts to verify:

(1) These choices contain no duplicate rows.

(2) The resulting minor has nonzero determinant.

The first is clear since $S_{i}$ and $S_{j}$ are disjoint for $i \neq j$ and each row associated to an element of $S_{k}$ has at most one entry containing a $c_{i}(k, k)$.

For the second, we start by examining the entries in each row. Each row associated to $\phi_{i}$ for $i \neq N$ contains a $c_{i}(N-2, N-2)\left(\right.$ or $c_{i}(N-1, N-1)$ for $N=3$ ) whose value depends on at least $c_{i}(N-3, N-3)$ (or $c_{i}(1,1)$ for $N=3)$ so is not identically 0 . In addition, each of these rows contains a corresponding

$$
c_{i}(N-2, N)= \begin{cases}-1-c_{i}(N-2, N-2) & \text { for } i=0, \\ 1-c_{i}(N-2, N-2) & \text { for } i=N-2, \\ -c_{i}(N-2, N-2) & \text { otherwise. }\end{cases}
$$

For $x_{1}^{2}$ we have $c_{0}(1,1)$ and $c_{0}(1, N)=-1-c_{0}(1,1)$. For $x_{0}^{2}$, we have $c_{1}(0,0)$ and $c_{1}(0, N)=-c_{0}(1,1)$. For $x_{2 k}^{2}$ with $k>0$, there is a $c_{2 k}(2 k, 2 k)$ and a 
$c_{2 k}(2 k, N)=1-c_{2 k}(2 k, 2 k)$. For $k>1$ there is a $c_{2 k}(2 k-1,2 k-1)$ and a $c_{2 k}(2 k-1, N)=1-c_{2 k}(2 k-1,2 k-1)$. The rest of the entries are either constants or depend on $c_{1}(0, N-1)$. Also note that each row contains each $c_{i}(k, k)$ at most once (in addition to the corresponding $c_{i}(k, N)$ ).

Note that $c_{i}(k, k)$ and $c_{i}(k, N)$ are possibly linearly dependent and that our choice of ordering has $c_{i}(k, k)$ appearing farther right in the matrix than $c_{i}(k, N)$. For the other entries, we are choosing one coefficient per iteration, so they are either constant, independent, or the next depends on the previous in a quadratic (or higher) fashion (since each $\phi_{i}$ is degree 2).

Assume that we have some linear combination of the rows that produces a row identically 0 . Each row contains a $c_{i}(k, k)$ on the diagonal for some $i$ and $k$ and a $c_{i}(k, N)$ in some other entry. For the linear combination to result in 0 , there are three cases to consider.

CASE 1. Assume two rows in the combination contain $c_{i}(k, k)$ and $c_{i}(k, N)$ in the same column. By our choice of ordering, the respective $c_{i}(k, N)$ and $c_{i}(k, k)$ in those rows would not be in the same column. Hence, we must also include rows in the combination that contain $c_{i}(k, N)$ and $c_{i}(k, k)$ in the corresponding columns. Again by our choice of ordering, we need to include at least two rows to do this and then we still have unpaired $c_{i}(k, k)$ and $c_{i}(k, N)$ as before. Therefore, we cannot choose any number of rows so that all of the $c_{i}(k, k)$ and $c_{i}(k, N)$ are paired by column.

CASE 2. Notice that by our choice of the $S_{i}$ we have guaranteed that we cannot have $c_{j}(N-2, N-2)$ and $c_{i}(k, k)$ in the same column for any $k \neq N-2$. Let $j$ be such that $c_{j}(N-2, N-2)$ is on the diagonal of the minor. Assume two rows in the combination have $c_{i}(N-2, N-2)$ and $c_{j}(N-2, N-2)$ in the same column for $i \neq j$. But with $c_{j}(N-2, N-2)$ used for $S_{N-2}$, the row containing $c_{i}(N-2, N-2)$ must also contain $c_{i}(k, k)$ for some $k \neq N-2$. As in Case 1 we are unable to find a combination of rows that pairs all of the $c_{i}(k, k)$ and $c_{i}(k, N)$.

CASE 3. Assume we have $c_{i}(k, k)$ and $c_{i}(k, N)$ paired with constants to get a combination of rows identically 0 . However, every row containing a $c_{i}(k, k)$ with $k \neq N-2$ also contains $c_{j}(N-2, N-2)$ for some $j$. These $c_{j}(N-2, N-2)$ must be paired either with constants or with other $c_{t}(N-2, N-2)$. However, they cannot be paired with constants since the $c_{i}(k, k)$ are already paired with constants in a combination that results in 0 , and $c_{i}(k, k)$ and $c_{j}(N-2, N-2)$ are not related in a linear fashion. Case 2 eliminates the possibility of pairing with another $c_{t}(N-2, N-2)$ for some $t$. So we must have $k=N-2$. Then all of the rows in the combination are associated to the same $\phi_{j}$, and hence entries in columns cannot be paired appropriately to result in a combination of 0 . 
Therefore, no linear combination can have all entries as 0 and the determinant of this minor is not identically 0 . Therefore, there are infinitely many choices of the coefficients that produce a map that is a morphism.

For the families with a periodic point with smaller primitive period, the matrix is similar but with more free constants, so similar choices of rows will also produce a minor with nonzero determinant.

\section{Proof of Theorem 3}

Lemma 2. Given $\phi_{1}: \mathbb{P}^{N} \rightarrow \mathbb{P}^{N}$ a polynomial morphism with a point $P_{1}$ of primitive period $n$, and $\phi_{2}: \mathbb{P}^{M} \rightarrow \mathbb{P}^{M}$ a polynomial morphism with $P_{2}$ of primitive period $m$, there exists a polynomial morphism $\psi: \mathbb{P}^{N+M} \rightarrow \mathbb{P}^{N+M}$ and a point $P$ with primitive period $\operatorname{lcm}(n, m)$.

Proof. We restrict $\phi_{1}$ to the affine chart $\mathbb{A}^{N}$ with $x_{N} \neq 0$ and $\phi_{2}$ to the affine chart $\mathbb{A}^{M}$ with $x_{M} \neq 0$. The restricted points $\widetilde{P_{1}}$ and $\widetilde{P_{2}}$ still have period $n$ and $m$, and the product map $\widetilde{\phi_{1}} \times \widetilde{\phi_{2}}: \mathbb{A}^{N+M} \rightarrow \mathbb{A}^{N+M}$ has the product of the dehomogenizations $\widetilde{P}=\left(\widetilde{P_{1}}, \widetilde{P_{2}}\right)$ as a periodic point of primitive period $\operatorname{lcm}(n, m)$. This fact is simply the statement that the product of a cyclic group of order $n$ with a cyclic group of order $m$ has order $\operatorname{lcm}(n, m)$.

Now, homogenizing $\widetilde{\phi_{1}} \times \widetilde{\phi_{2}}$ to a map $\psi: \mathbb{P}^{N+M} \rightarrow \mathbb{P}^{N+M}$ we know that the first $N$ forms and $x_{N+M}^{2}$ have no common nontrivial zeros in $x_{0}, \ldots, x_{N-1}, x_{N+M}$ and the next $M$ forms and $x_{N+M}^{2}$ have no common nontrivial zeros in $x_{N}, \ldots, x_{N+M}$. Since the only variable shared between the two sets of forms is $x_{N+M}$, the map $\psi$ is also a morphism and the homogenization of $\widetilde{P}$ has primitive period $\operatorname{lcm}(n, m)$ for $\psi$.

Proof of Theorem 3 . From Theorems 1 and 2 we can find morphisms $\phi: \mathbb{P}^{N} \rightarrow \mathbb{P}^{N}$ with $\mathbb{Q}$-rational periodic points with primitive period $1,2, \ldots$, $(N+1)(N+2) / 2$. Fix $s$ a positive integer. Let $M=\lfloor N / s\rfloor$. Then $(M+1)(M+2) / 2>(N / s)(N / s) / 2=N^{2} / 2 s^{2}$ and for every prime $p \leq$ $N^{2} / 2 s^{2}$ there is a point with primitive period $p$ for some polynomial morphism of $\mathbb{P}^{M}$. Fix $\epsilon>0$ and choose $N$ large enough that the interval $\left((1-\epsilon) N^{2} / 2 s^{2}, N^{2} / 2 s^{2}\right)$ has at least $s$ primes $p_{1}, \ldots, p_{s}$. We apply Lemma 2 to combine these points and associated morphisms to get a point $P \in \mathbb{P}^{s M}$ $=\mathbb{P}^{N}$, which has primitive period

$$
p_{1} \cdots p_{s} \geq \frac{(1-\epsilon)^{s}}{2^{s} s^{2 s}} N^{2 s}
$$

for a polynomial morphism $\psi: \mathbb{P}^{N} \rightarrow \mathbb{P}^{N}$.

5. Some examples with larger primitive periods. With slightly different choices of coefficients, it is occasionally possible to increase the 
primitive period by more than 2 with a choice of a single set of coefficients. While a general method to ensure this occurrence was not discovered, in practice it is possible to construct a polynomial map for a specific $N$ with a periodic point with primitive period that exceeds the bound presented in Theorem 1. These can then be combined as in Lemma 2 to produce morphisms of $\mathbb{P}^{N}$ with $\mathbb{Q}$-rational periodic points of large primitive period. The following examples present such maps for $N=2,3$, and 4 . For the reader's convenience, the following table shows the trivial lower bound $\left(\begin{array}{c}N+2 \\ 2\end{array}\right)$, the lower bound from Theorem 1, and the primitive period exhibited in the example of a polynomial morphism $\phi: \mathbb{P}^{N} \rightarrow \mathbb{P}^{N}$. Note that since we are dealing with maps on $\mathbb{P}^{N}$ outside of the scope of Theorem 2 , the maps were verified explicitly to be morphisms, but the details are omitted here.

\begin{tabular}{|c|c|c|c|}
\hline$N$ & Trivial bound & Theorem 1 bound & Example period \\
\hline 2 & 6 & 7 & 9 \\
\hline 3 & 10 & 11 & 24 \\
\hline 4 & 15 & 16 & 72 \\
\hline
\end{tabular}

ExAmple 1 . The point $[0,0,1] \in \mathbb{P}^{2}$ is a periodic point of primitive period 9 for the morphism

$$
\begin{aligned}
\phi\left(\left[x_{0}, x_{1}, x_{2}\right]\right)= & {\left[-38 / 45 x_{0}^{2}+\left(2 x_{1}-7 / 45 x_{2}\right) x_{0}+\left(-1 / 2 x_{1}^{2}-1 / 2 x_{2} x_{1}+x_{2}^{2}\right),\right.} \\
& \left.-67 / 90 x_{0}^{2}+\left(2 x_{1}+157 / 90 x_{2}\right) x_{0}-x_{2} x_{1}, x_{2}^{2}\right] .
\end{aligned}
$$

Example 2. The point $[0,0,0,1] \in \mathbb{P}^{3}$ is a periodic point of primitive period 24 for the morphism

$$
\begin{aligned}
\phi\left(\left[x_{0}, x_{1}, x_{2}, x_{3}\right]\right)= & {\left[\left(-x_{1}-x_{3}\right) x_{0}+\left(-13 / 30 x_{1}^{2}+13 / 30 x_{3} x_{1}+x_{3}^{2}\right),\right.} \\
& -1 / 2 x_{0}^{2}+\left(-x_{1}+3 / 2 x_{3}\right) x_{0}+\left(-1 / 3 x_{1}^{2}+4 / 3 x_{3} x_{1}\right), \\
& \left.-3 / 2 x_{2}^{2}+5 / 2 x_{2} x_{3}+x_{3}^{2}, x_{3}^{2}\right]
\end{aligned}
$$

created by combining a periodic point of primitive period 8 in $\mathbb{P}^{2}$ and a periodic point of primitive period 3 in $\mathbb{P}^{1}$.

EXAmple 3 . The point $[0,0,0,0,1] \in \mathbb{P}^{4}$ is a periodic point of primitive period 72 for the morphism

$$
\begin{aligned}
\phi\left(\left[x_{0}, x_{1},\right.\right. & \left.\left.x_{2}, x_{3}, x_{4}\right]\right) \\
= & {\left[-38 / 45 x_{0}^{2}+\left(2 x_{1}-7 / 45 x_{4}\right) x_{0}+\left(-1 / 2 x_{1}^{2}-1 / 2 x_{4} x_{1}+x_{4}^{2}\right),\right.} \\
& -67 / 90 x_{0}^{2}+\left(2 x_{1}+157 / 90 x_{4}\right) x_{0}-x_{4} x_{1}, \\
& \left(-x_{3}-x_{4}\right) x_{2}+\left(-13 / 30 x_{3}^{2}+13 / 30 x_{4} x_{3}+x_{4}^{2}\right), \\
& \left.-1 / 2 x_{2}^{2}+\left(-x_{3}+3 / 2 x_{4}\right) x_{2}+\left(-1 / 3 x_{3}^{2}+4 / 3 x_{4} x_{3}\right), x_{4}^{2}\right]
\end{aligned}
$$

created by combining periodic points of primitive period 8 and 9 in $\mathbb{P}^{2}$. 


\section{References}

[1] E. Flynn, B. Poonen, and E. Schaefer, Cycles of quadratic polynomials and rational points on a genus-2 curve, Duke Math. J. 90 (1997), 435-463.

[2] F. Macaulay, The Algebraic Theory of Modular Systems, Cambridge Univ. Press, Cambridge, 1916; reprinted, 1994.

[3] M. Manes, $\mathbb{Q}$-rational cycles for degree-2 rational maps having an automorphism, Proc. London Math. Soc. 96 (2008), 669-696.

[4] P. Morton, Arithmetic properties of periodic points of quadratic maps, Acta Arith. 62 (1992), 343-372.

[5] D. Northcott, Periodic points on an algebraic variety, Ann. of Math. 51 (1950), $167-177$.

[6] M. Stoll, Independence of rational points on twists of a given curve, Compos. Math. 142 (2006), 1201-1214.

Benjamin Hutz

Department of Mathematics and Computer Science

Amherst College, P.O. 5000

Amherst, MA 01002-5000, U.S.A.

E-mail: bhutz@amherst.edu

Received on 12.12.2008

and in revised form on 26.6.2009 